Commun. Math. Phys. 148, 117-137 (1992)

Communications in

Mathematical

Physics

(C) Springer-Verlag 1992

\title{
Algebraic Structure of Topological Superconformal Field Theory on Riemann Surfaces
}

\author{
Abbas Ali, Alok Kumar, Jnanadeva Maharana and Gautam Sengupta \\ Institute of Physics, Bhubaneswar-751005, India
}

Received July 15, 1991; in revised form January 28, 1992

\begin{abstract}
The algebraic structure of a topological superconformal field theory on a compact Riemann surface is investigated. The Krichever-Novikov [K-N] global operator formalism is used to obtain an $N=4$ super $\mathrm{K}-\mathrm{N}$ algebra on a Riemann surface. Subsequently this $N=4$ algebra is shown to possess an $N=3 \mathrm{~K}-\mathrm{N}$ subalgebra. The $N=3$ subalgebra is then twisted to derive a topological version of the Krichever-Novikov algebra with a residual $N=2$ superconformal structure. The BRST charge of the associated topological field theory on the Riemann surface is shown to be genus dependent in this formalism and the global generalization of the BRST derivative conditions are obtained. The complete BRST structure of the theory is explicitly elucidated.
\end{abstract}

\section{Introduction}

Recently topological field theories have assumed considerable significance in the context of the nonperturbative aspects of string theories. It was earlier shown by Witten [1] that these theories may be constructed by twisting an $N=2$ supersymmetric non-linear sigma model. Following this line of investigation Eguchi and Yang [2] constructed topological conformal field theories in two dimensions by twisting an $N=2$ superconformal field theory (SCFT)[3]. Furthermore, it was shown in later investigations that the minimal versions [4] of these models, when coupled to two dimensional topological gravity [5], are equivalent to the matrix model $[6,7]$ description of non-critical string theories. In addition, the supersymmetric generalizations of the gravity sector have also been investigated [8].

The generators of an $N=2$ SCFT [3] are: a weight two energy momentum tensor $T(z)$, two supercharges $G^{ \pm}(z)$ of weight $\frac{3}{2}$, and a $U(1)$ current $J(z)$ of weight 1. In ref. [2] it was observed that upon twisting the energy momentum tensor of these theories via the $U(1)$ current, a centerless conformal algebra with a background charge is obtained. Moreover the two supercharges are transformed to a weight two and a weight one operator with respect to the redefined energy momentum tensor. In addition, the new operator of dimension 1 now plays the role of a BRST current such that the BRST charge obtained from it is nilpotent and the 
twisted energy momentum tensor is a BRST derivative of the dimension two operator. This defines the algebraic structure of a topological conformal field theory (TCFT). The physical subspace of the Hilbert space of this theory is a cohomology of this algebra.

It was shown in ref. [9] that a topological superconformal field theory (TSCFT) may be obtained starting from an $N=3 \mathrm{SCFT}[3,10]$. Moreover a $N=2$ TSCFT was obtained [11] starting from an $N=4$ SCFT of Sevrin et al. [12].

However the structure of these topological field theories on a Riemann surface have not been explicitly investigated. In an earlier work, we have addressed this important question in the framework of the Krichever-Novikov global operator formalism $[13,14,15]$ for conformal field theories on a compact Riemann surface with two distinguished punctures. In this article we propose to generalize our analysis to the investigation of the algebraic structure of an $N=2$ TSCFT on such a Riemann surface starting from an $N=4$ SCFT in the $\mathrm{K}-\mathrm{N}$ framework. We find an interesting, global generalization of the algebraic structure of the $N=2$ TSCFT on the complex plane [11]. Furthermore we obtain a genus dependent BRST charge on the Riemann surface such that all the generators in the theory are accompanied by a BRST partner. The BRST derivative condition is modified in addition to a generalized BRST derivative on the Riemann surface.

The rest of the article is organized as follows. In Sect. II we present a resume of our construction of a TCFT [16] starting from an $N=2$ SCFT on a Riemann surface [17]. In the next section (III) we explicitly construct the $N=4 \mathrm{~K}-\mathrm{N}$ superalgebra on a Riemann surface. In Sect. IV we define some of the generators of this theory to extract an $N=3 \mathrm{~K}-\mathrm{N}$ substructure and show that the remaining generators organize into a weight zero superfield of the $N=3$ supersymmetry. In the next section (V) we twist this $N=3$ theory to obtain an $N=2$ TSCFT and show that the remaining generators form a weight zero superfield of the $N=2$ supersymmetry. We also explicitly show the full BRST structure of the resulting TCFT in Sect. VI. In the last section (VII) we present a summary of our investigation and a discussion of the results obtained. In addition, there is an Appendix containing the explicit global forms of the structure constants for the various algebras.

\section{Krichever-Novikov Formulation of Topological Conformal Field Theory}

In this section we briefly outline the essential features of the $\mathrm{K}-\mathrm{N}$ operator formalism $[13,14,15]$ and describe our construction of a TCFT [16] by twisting an $N=2$ SCFT [17] on a Riemann surface $\Sigma_{g}$ of genus $g$. We choose $\Sigma_{g}$ with $g>1$ and having two distinguished punctures $P_{ \pm}$corresponding to the points $z=0$ and $z=\infty$ on the complex plane. K-N [13] provided a complete orthonormal set of Fourier Laurent bases for the space of meromorphic forms of weight $\lambda$ on $\Sigma_{g}$, holomorphic away from the punctures where they might posess singularities. This was done through the application of the Riemann-Roch theorem which ensures the existence of the basis for the space of meromorphic forms of weight $\lambda$. In their analysis, the classical form of the Riemann-Roch theorem in terms of divisors on a compact Riemann surface was transformed to the theorem in terms of meromorphic sections of tensor products for $\Lambda^{\lambda}$, where $\Lambda^{\lambda}$ is the canonical divisor corresponding to the canonical line bundle on $\Sigma_{g}$. The section of each line bundle of this tensor product has prescribed orders of poles at $P_{ \pm}$and corresponding zeros 
elsewhere. The details of the construction may be found in $\mathrm{K}-\mathrm{N}$ [13] and Bonora et al. [14]. The asymptotic forms for these basis in a local complex co-ordinate system $z_{ \pm}$around $P_{ \pm}$are given as follows:

For $\lambda \in Z$ and $\lambda \neq 0,1$,

$$
f_{j}^{\lambda}=\phi_{j}^{(\lambda) \pm} z_{ \pm}^{ \pm j-s(\lambda)}\left[1+O\left(z_{ \pm}\right)\right]\left(d z_{ \pm}\right)^{\lambda},
$$

where $s(\lambda)=\frac{g}{2}-\lambda(g-1)$ and $\phi_{j}^{(\lambda) \pm}$ are normalization constants. The index $j$ runs over $\cdots-\frac{g}{2},-\frac{g}{2}+1, \ldots$ and is integer or half integer depending on the parity of g. It can be observed that the order of the poles as prescribed by $\mathrm{K}-\mathrm{N}$ [13] is present in the expression for the asymptotic forms.

For $\lambda=0$, we have the basis for the space of meromorphic functions $A_{n}(P), P$ being a generic point on $\Sigma_{g}$, with the asymptotic forms:

$$
A_{n}\left(z_{ \pm}\right)=a_{n}^{ \pm} z_{ \pm}^{( \pm n-g / 2)}\left[1+O\left(z_{ \pm}\right)\right] \quad|n| \geqq \frac{g}{2}+1
$$

and

$$
\begin{aligned}
A_{n}\left(z_{ \pm}\right) & =a_{n}^{ \pm} z_{ \pm}^{( \pm n-g / 2 \pm 1 / 2-\varepsilon)}\left[1+O\left(z_{ \pm}\right)\right] \quad|n|<\frac{g}{2}, \\
A_{g / 2} & =1,
\end{aligned}
$$

where $\varepsilon=\frac{1}{2}$, and $a_{n}^{+}=1$. These expressions are the consequence of the Weirstrass gap theorem which states that the meromorphic functions having poles of order $p$ at a given point on $\Sigma_{g}$ cannot be extended holomorphically outside it for $g$ values of $p$.

For $\lambda=1$, we have the basis for one forms $W_{j}(P)$ on $\Sigma_{g}$. Their asymptotic forms around $P_{ \pm}$is given in the local co-ordinate system $z_{ \pm}$as,

$$
\begin{aligned}
& W_{j}\left(z_{ \pm}\right)=\beta_{j}^{ \pm} z_{ \pm}^{(\mp j+g / 2 \mp 1 / 2-\varepsilon)}\left[1+O\left(z_{ \pm}\right)\right] d z_{ \pm} \quad \text { for }|j|<\frac{g}{2} \quad n \neq \frac{g}{2} \\
& W_{j}\left(z_{ \pm}\right)=f_{-j}^{1}\left(z_{ \pm}\right) \quad|j| \geqq \frac{g}{2}+1
\end{aligned}
$$

and

$$
W_{g / 2}(P)=d k(P),
$$

where $d k(P)$ is an abelian differential of the third kind on the Riemann surface $\Sigma_{g}$, having simple poles at the two punctures $P_{ \pm}$and residues \pm 1 and normalized such that the periods over all cycles are imaginary. $d k(P)$ defined thus provides a basis for holomorphic differentials on $\Sigma_{g}$ which may be written as a linear combination of the canonical homology basis.

When $\lambda \in \mathbf{Z}+\frac{1}{2}$, the basis may be constructed by taking the meromorphic sections of the tensor products of $\Lambda^{\lambda}$ with a given spin structure. There are two different cases,

(i) The space of forms holomorphic outside $P_{ \pm}$and a slit from $P_{+}$to $P_{-}$along a Jordan curve. This corresponds to the Ramond (R) sector.

(ii) The space of forms holomorphic outside $P_{ \pm}$. This corresponds to the Neveu-Schwarz (N-S) sector. 
We will restrict our consideration to the $\mathrm{N}-\mathrm{S}$ sector only as this is the sector relevant for the TCFT obtained after twisting. The asymptotic form for these bases are as follows:

$$
f_{\alpha}^{\lambda}(z \pm)=\psi^{ \pm} z_{ \pm}^{\alpha-s(\lambda)}\left[1+O\left(z_{ \pm}\right)\right]\left(d z_{ \pm}\right)^{\lambda},
$$

where $\alpha \in \mathbf{Z}+\frac{1}{2}$ for the $\mathrm{N}-\mathrm{S}$ sector.

This formalism provides for an elegant parametrization of the Riemann surface by a set of level curves $C_{\tau}$ of constant $\tau$ analogous to the equal time circles on the complex plane. The parameter $\tau$ plays the role of a global univalent and unique time parameter on $\Sigma_{g}$ in string theoretic applications. This is defined in the K-N formalism as,

$$
\tau(P)=\operatorname{Re} \int_{P_{0}}^{P} d k(P),
$$

where $d k(P)$ is as given earlier, and $P_{0}$ is some reference point on $\Sigma_{g}$. As $P \rightarrow P_{ \pm}$the contours $C_{\tau} \rightarrow C_{ \pm}$and $\tau \rightarrow \mp \infty$, where $C_{ \pm}$are circles in the local co-ordinate system $z_{ \pm}$.

As an illustration of the operator formalism outlined above we present a brief summary of our earlier investigation of the construction of a TCFT on $\Sigma_{g}$ from an $N=2$ SCFT. The generators of the $N=2$ SCFT on $\Sigma_{g}$ are now meromorphic tensors of definite weight on $\Sigma_{g}$, given by a weight two energy momentum tensor $T(P)$, two supercharges $G^{ \pm}(P)$ of weight $\frac{3}{2}$, and a $U(1)$ current $J(P)$ of weight one. The bases described earlier may be used to perform mode expansion of these operators on $\Sigma_{g}$ as follows:

$$
\begin{aligned}
T(P) & =\sum_{n} L_{n} \Omega_{n}(P), \\
G^{ \pm}(P) & =\sum_{\alpha} G_{\alpha}^{ \pm} B_{\alpha}(P), \\
J(P) & =\sum_{k} J_{k} W_{k}(P) .
\end{aligned}
$$

The energy momentum tensor on $\Sigma_{g}$ is then twisted as,

$$
\tilde{T}(P)=T(P)+\frac{1}{2} d_{P} J(P),
$$

where $d_{P}$ is a global derivative on $\Sigma_{g}$ having the local form $\left(\partial_{z_{ \pm}} d z_{ \pm}\right)$in the co-ordinate system $z_{ \pm}$as defined earlier. As $P \rightarrow P_{ \pm}$, we recover the expression for the twisted energy momentum tensor on the complex plane in the local co-ordinate system $z_{ \pm}$. This is given as [2],

$$
\tilde{T}\left(z_{ \pm}\right)=T\left(z_{ \pm}\right)+\frac{1}{2} \partial_{z_{ \pm}} J\left(z_{ \pm}\right) \text {. }
$$

It can be easily verified that with respect to the redefined energy momentum tensor Eq. (2.14), the Virasoro part of the superconformal algebra is centerless and the supercharges $G^{ \pm}(z)$ transform as a dimension one and a dimension two field respectively. This is evident from the operator product expansions for the twisted 
Table 1. The $\mathrm{K}-\mathrm{N}$ bases and their duality and completeness relations

\begin{tabular}{llll}
\hline$\lambda$ & Basis & Dual basis & $\lambda$ \\
\hline-1 & $e_{m}(P)$ & $\Omega_{n}(P)$ & 2 \\
0 & $A_{m}(P)$ & $W_{n}(P)$ & 1 \\
$-1 / 2$ & $C_{\alpha}(P)$ & $B_{\beta}(P)$ & $3 / 2$ \\
$1 / 2$ & $g_{\alpha}(P)$ & $g_{\beta}(P)$ & $1 / 2$ \\
\hline
\end{tabular}

Duality and Completeness

$$
\begin{array}{ll}
\frac{1}{2 \pi i} \oint_{C_{\tau}} e_{m}(P) \Omega_{n}(P)=\delta_{m n} & \frac{1}{2 \pi i} \sum_{m} e_{m}(P) \Omega_{m}(\tilde{P})=D_{\tau}(P, \tilde{P}) \\
\frac{1}{2 \pi i} \oint_{C_{\tau}} A_{m}(P) W_{n}(P)=\delta_{m n} & \frac{1}{2 \pi i} \sum_{m} A_{m}(P) W_{m}(\tilde{P})=\Delta_{\tau}(P, \tilde{P}) \\
\frac{1}{2 \pi i} \oint_{C_{\tau}} C_{\alpha}(P) B_{\beta}(P)=\delta_{\alpha \beta} & \frac{1}{2 \pi i} \sum_{\alpha} C_{\alpha}(P) B_{\alpha}(\tilde{P})=d_{\tau}(P, \tilde{P}) \\
\frac{1}{2 \pi i} \oint_{C_{\tau}} g_{\alpha}(P) g_{-\beta}(P)=\delta_{\alpha \beta} & \frac{1}{2 \pi i} \sum_{\alpha} g_{\alpha}(P) g_{-\alpha}(\tilde{P})=\delta_{\tau}(P, \tilde{P})
\end{array}
$$

version of the theory which are presented in Appendix A. Consequently these charges now have new mode expansions. These are,

$$
\begin{aligned}
& \tilde{G}(P)=\sum_{m} \tilde{G}_{m} \Omega_{m}(P), \\
& \tilde{Q}(P)=\sum_{m} \tilde{Q}_{m} W_{m}(P),
\end{aligned}
$$

where $\tilde{G}(P)$, and $\tilde{Q}(P)$ denotes the modified operators in the twisted theory. It is well known that although the OPE on $\Sigma_{g}$ are in general $g$ dependent, their singularity structures are genus independent and the same as those on the complex plane. So we can use the standard OPE together with the $\mathrm{K}-\mathrm{N}$ bases in Table 1 to obtain the topological version of the $\mathrm{K}-\mathrm{N}$ algebra. The mode expansion Eqs. (2.7, $2.9,2.12,2.13)$ are inverted using the duality properties of the $\mathrm{K}-\mathrm{N}$ bases to obtain the Fourier projections,

$$
\begin{aligned}
& \tilde{L}_{j}=\frac{1}{2 \pi i} \oint_{P \in C_{\tau}} \tilde{T}(P) e_{j}(P), \\
& \tilde{G}_{j}=\frac{1}{2 \pi i} \oint_{P \in C_{\tau}} \tilde{G}(P) e_{j}(P), \\
& \tilde{Q}_{j}=\frac{1}{2 \pi i} \oint_{P \in C_{\tau}} \tilde{Q}(P) A_{j}(P), \\
& J_{k}=\frac{1}{2 \pi i} \oint_{P \in C_{r}} J(P) A_{k}(P) .
\end{aligned}
$$

These equations may now be expressed in the local cordinate system $z$ around the puncture $P_{+}$. Knowing the OPE of the generators in the twisted theory which 
may be evaluated from those in the $N=2$ SCFT we can construct the algebra by means of the complex contour representation and we arrive at the following topological $\mathrm{K}-\mathrm{N}$ algebra:

$$
\begin{gathered}
{\left[\tilde{L_{m}}, \tilde{L_{n}}\right]=\sum_{s=-g_{0}}^{g_{0}} A_{m n}^{s} \tilde{L}_{m+n-s},} \\
{\left[\tilde{L}_{m}, \tilde{G}_{n}\right]=\sum_{s=-g_{0}}^{g_{0}} A_{m n}^{s} \tilde{G}_{m+n-s},} \\
{\left[\tilde{L}_{m}, \tilde{Q}_{n}\right]=-\sum_{s=-g_{0}}^{g_{0}} C_{m n}^{s} \tilde{Q}_{m+n-s},} \\
\left\{\tilde{G}_{m}, \tilde{Q}_{n}\right\}=\sum_{s=-g / 2}^{g / 2} P_{m n}^{s} \tilde{L}_{m+n-s}+\sum_{s=-g_{0}}^{g_{0}} E_{m n}^{s} J_{m+n-s}+\frac{c}{3} \Pi_{m n}, \\
{\left[\tilde{L}_{m}, J_{n}\right]=-\sum_{s=-g_{0}}^{g_{0}} C_{m n}^{s} J_{m+n-s}+\frac{c}{6} \sum_{j} t_{j m} K_{j n},} \\
{\left[J_{m}, \tilde{G}_{n}\right]=\sum_{s=-g / 2}^{g / 2} D_{m n}^{s} \tilde{G}_{m+n-s},} \\
{\left[J_{m}, \tilde{\left.Q_{n}\right]}=\sum_{s=-g / 2}^{g / 2} B_{m n}^{s} \tilde{Q}_{m+n-s},\right.} \\
{\left[J_{m}, J_{n}\right]=\frac{c}{3} K_{m n},} \\
\left\{\tilde{G}_{m}, \tilde{G}_{n}\right\}=\left\{\tilde{Q_{m}}, \tilde{Q}_{n}\right\}=0,
\end{gathered}
$$

where the structure constants of the algebra are given explicitly in the Appendix.

The BRST charge in the twisted version of the theory is defined as an integral of the new, dimension one operator over a closed cycle on $\Sigma_{g}$. This may be chosen as one of the level curves $C_{\tau}$. Explicitly, we have

$$
Q_{B}=\oint_{P \in C_{\mathrm{r}}} \tilde{Q}(P)
$$

With the use of the appropriate mode expansion and the asymptotic forms for the bases in the local complex co-ordinate system $z_{ \pm}$around the punctures $P_{ \pm}$we have on $\Sigma_{g}$,

$$
Q_{B}=\tilde{Q}_{g / 2}
$$

Equation (2.24) then gives the relation

$$
\left\{\tilde{G}_{m}, \tilde{Q}_{B}\right\}=\sum_{s=-g / 2}^{g / 2} P_{m(g / 2)}^{s} \tilde{L}_{m+g / 2-s}
$$

as the structure constants in the other terms vanish for $n=g / 2$. This is a global generalization of the BRST derivative condition for the energy momentum tensor 
on the complex plane $[2,4]$. We remark here that on $\Sigma_{g}, \tilde{T}(P)$ is a generalized BRST derivative of the generator $\tilde{G}(P)$. This reduces to the familiar relation $[2,4]$ on the complex plane when evaluated in a suitable local co-ordinate system. Notice that on $\Sigma_{g}$ the BRST charge is genus dependent. This naturally gives a genus dependent physical state cohomology in the Hilbert space of the associated TCFT on $\Sigma_{g}$. It has been shown in ref. [16] that the physical subspace consists of those states which are equivalent up to a BRST exact state to the chiral primary states of the untwisted theory. It was observed that all these states have zero weight with respect to the redefined modes of the energy momentum tensor. This completes a resume of our construction of a TCFT on $\Sigma_{g}$ from an $N=2$ SCFT. We now consider the application of this formalism to investigate the algebraic structure of an $N=2$ TSCFT derived from an $N=4$ SCFT on $\Sigma_{g}$. In the next section we present the explicit construction of an $N=4 \mathrm{~K}-\mathrm{N}$ superalgebra on $\Sigma_{g}$.

\section{III. $N=4$ Krichever-Novikov Superalgebra}

To construct an $N=4$ super $\mathrm{K}-\mathrm{N}$ algebra on $\Sigma_{g}$ we follow the procedure outlined in Sect. II. The generators of this algebra are given by the weight two energy momentum tensor $T(P)$, four weight $\frac{3}{2}$ supercurrents $G^{a}(P)$, six generators $D^{ \pm i}(P)$ of the $S U(2) \times S U(2)$ Kac-Moody algebra, four weight $\frac{1}{2}$ fields $Q^{a}(P)$ and a $U(1)$ generator $U(P)$. These generators may be mode expanded in the appropriate $\mathrm{K}-\mathrm{N}$ bases as follows:

$$
\begin{aligned}
T(P) & =\sum_{n} L_{n} \Omega_{n}(P), \\
G^{a}(P) & =\sum_{\alpha} G_{\alpha}^{a} B_{\alpha}, \\
D^{ \pm i}(P) & =\sum_{m} D_{m}^{ \pm i} W_{m}(P), \\
Q^{a}(P) & =\sum_{\beta} Q_{\beta}^{a} g_{\beta}(P), \\
U(P) & =\sum_{m} U_{m} W_{m}(P),
\end{aligned}
$$

where $i=1$ to 3 and $a=1$ to 4 . The Eqs. (3.1), (3.5) may now be inverted to give the following Fourier projections:

$$
\begin{aligned}
L_{n} & =\frac{1}{2 \pi i} \oint_{P \in C_{\tau}} e_{n}(P) T(P), \\
G_{\alpha}^{a} & =\frac{1}{2 \pi i} \oint_{P \in C_{\tau}} C_{\alpha}(P) G^{a}(P), \\
D_{m}^{ \pm i} & =\frac{1}{2 \pi i} \oint_{P \in C_{\tau}} A_{m}(P) D^{ \pm i}(P), \\
Q_{\beta}^{a} & =\frac{1}{2 \pi i} \oint_{P \in C_{\tau}} g_{-\beta}(P) Q^{a}(P), \\
U_{m} & =\frac{1}{2 \pi i} \underset{P \in C_{\tau}}{\oint_{m} A_{m}(P) U(P),}
\end{aligned}
$$


The $N=4 \mathrm{~K}-\mathrm{N}$ algebra is then derived in an analogous fashion as in Sect. II by expressing the algebra as double contour integrals in the local co-ordinate system around the puncture $P_{ \pm}$. The OPE for the generators are standard and using these to evaluate the contour integrals we arrive at the $N=4$ super $\mathrm{K}-\mathrm{N}$ algebra. Explicitly we have for the modes of the energy momentum tensor,

$$
\begin{aligned}
{\left[L_{m}, L_{n}\right] } & =\sum_{s=-g_{0}}^{g_{0}} A_{m n}^{s} L_{m+n-s}+\frac{c}{12} \chi_{m n}, \\
{\left[L_{m}, G_{\alpha}^{a}\right] } & =\sum_{s=-g_{0}}^{g_{0}} V_{m \alpha}^{s} G_{m+\alpha-s}^{a}, \\
{\left[L_{m}, D_{n}^{ \pm i}\right] } & =\sum_{s=-g_{0}}^{g_{0}} C_{m n}^{s} D_{m+n-s}^{ \pm i}, \\
{\left[L_{m}, U_{n}\right] } & =\sum_{s=-g_{0}}^{g_{0}} C_{m n}^{s} U_{m+n-s}, \\
{\left[L_{m}, Q_{\beta}^{a}\right] } & =\sum_{s=-g_{0}}^{g_{0}} X_{m \alpha}^{s} Q_{m+\alpha-s}^{a} .
\end{aligned}
$$

The algebra involving the supercharges and the weight $\frac{1}{2}$ generators are

$$
\begin{aligned}
\left\{G_{\alpha}^{a}, G_{\beta}^{a}\right\} & =\sum_{s=-g_{0}}^{g_{0}} \mathscr{E}_{\alpha \beta}^{s} M_{\alpha+\beta-s}^{a b}+2 \delta^{a b} \sum_{s=-g / 2}^{g / 2} F_{\alpha \beta}^{s} L_{\alpha+\beta-s}+\frac{c}{3} \mathscr{Q}_{\alpha \beta}, \\
M_{\alpha+\beta-s}^{a b} & =-4\left[\gamma \alpha_{a b}^{+i} D_{\alpha+\beta-s}^{+i}+(1-\gamma) \alpha_{a b}^{+i} D_{\alpha+\beta-s}^{-i}\right] \\
{\left[D_{m}^{+i}, G_{\alpha}^{a}\right] } & =\alpha_{a b}^{+i}\left[\sum_{s=-g / 2}^{g / 2} Y_{m \alpha}^{s} G_{m+\alpha-s}^{b}-2(1-\gamma) \sum_{s=-g_{0}}^{g_{0}} Z_{m \alpha}^{s} Q_{m+\alpha-s}^{b}\right] \\
{\left[D_{m}^{-i}, G_{\alpha}^{a}\right] } & =\alpha_{a b}^{-i}\left[\sum_{s=-g / 2}^{g / 2} Y_{m \alpha}^{s} G_{m+\alpha-s}^{b}+2 \gamma \sum_{s=-g_{0}}^{g_{0}} Z_{m \alpha}^{s} Q_{m+\alpha-s}^{b}\right] \\
\left\{Q_{\alpha}^{a}, G_{\beta}^{b}\right\} & =2 \sum_{s=-g / 2}^{g / 2} \mathscr{K}_{\alpha \beta}^{s}\left[\alpha_{a b}^{+i} D_{\alpha+\beta-s}^{+i}-\alpha_{a b}^{-i} D_{\alpha+\beta-s}^{-i}+\delta^{a b} U_{\alpha+b-s}\right] \\
\left\{Q_{\alpha}^{a}, Q_{\beta}^{b}\right\} & =-\tilde{c} \delta^{a b} \mathscr{P}_{\alpha \beta} .
\end{aligned}
$$

The modes of the Kac-Moody generators satisfy,

$$
\begin{aligned}
{\left[D_{m}^{ \pm i}, D_{n}^{ \pm j}\right] } & =\varepsilon^{i j k} \sum_{s=-g / 2}^{g / 2} I_{m n}^{s} D_{m+n-s}^{ \pm k}-\frac{k^{ \pm}}{2} \delta^{i j} J_{m n} \\
{\left[D_{m}^{ \pm i}, Q_{\alpha}^{a}\right] } & =\alpha_{a b}^{ \pm i} \sum_{s=-g / 2}^{g / 2} \mathscr{L}_{m \alpha}^{s} Q_{m+\alpha-s}^{b} .
\end{aligned}
$$


Finally the modes of the $U(1)$ current give

$$
\begin{aligned}
{\left[U_{m}, Q_{\alpha}^{a}\right] } & =0, \\
{\left[U_{m}, G_{\alpha}^{a}\right] } & =\sum_{s=-g_{0}}^{g_{0}} N_{m \alpha}^{s} Q_{m+\alpha-s}^{a}, \\
{\left[U_{m}, D_{n}^{ \pm i}\right] } & =0
\end{aligned}
$$

where $\gamma$ parametrizes the unitary representations of the $N=4$ superconformal algebra. The structure constants are presented explicitly in their global forms in the Appendix. This completes our construction of the $N=4$ superconformal algebra on $\Sigma_{g}$. In the next section we extract the $N=3$ subalgebra through suitable redefinitions of some of the generators and show that the remaining generators organize into a weight zero superfield with respect to the $N=3$ superconformal generators.

\section{The $N=3$ Substructure}

We redefine the generators of the $N=4$ superconformal algebra of the last section in the following fashion:

$$
\begin{aligned}
\tilde{T}(P) & =T(P)-\frac{1}{2}(2 \gamma-1) d_{P} U(P), \\
\left(J_{L}\right)^{i}(P) & =i\left[D^{+i}(P)+D^{-i}(P)\right], \\
\Gamma(P) & =-i Q^{4}(P), \\
\tilde{G}^{i}(P) & =G^{i}(P)-(2 \gamma-1) d_{P} Q^{i}(P) .
\end{aligned}
$$

The mode expansions for these generators in terms of the $\mathrm{K}-\mathrm{N}$ bases is obvious from Table 1 and we do not present them here explicitly. Following the procedure outlined in Sect. II we can express these redefined generators in the local $z$ coordinate system around the punctures $P_{ \pm}$. The Fourier projections for the generators may be derived using the duality relations between the bases in Table 1,

$$
\begin{aligned}
\tilde{L}_{m} & =\frac{1}{2 \pi i} \oint_{P \in C_{\tau}} \tilde{T}(P) e_{m}(P), \\
\tilde{G}_{\alpha}^{i} & =\frac{1}{2 \pi i} \oint_{P \in C_{\tau}} \tilde{G}^{i}(P) C_{\alpha}(P), \\
\Gamma_{\alpha} & =\frac{1}{2 \pi i} \oint_{P \in C_{\tau}} \Gamma(P) g_{-\alpha}(P), \\
\left(J_{L}^{i}\right)_{m} & =\frac{1}{2 \pi i} \oint_{P \in C_{\tau}} J_{L}^{i}(P) A_{m}(P) .
\end{aligned}
$$


We now follow exactly the same procedure as in Sects. II and III to express the algebra as double contour integrals using the local form of Eqs. (4.5)-(4.8) in the $z$ co-ordinate system around the punctures. The OPE for the modified generators may be derived knowing the OPE for the original theory. These OPE may be used in conjunction with the local form of the $\mathrm{K}-\mathrm{N}$ bases to derive the $N=3 \mathrm{~K}-\mathrm{N}$ subalgebra. For the modes of the new energy momentum tensor we have,

$$
\begin{aligned}
{\left[\tilde{L_{m}}, \tilde{L_{n}}\right] } & =\sum_{s=-g_{0}}^{g_{0}} A_{m n}^{s} \tilde{L}_{m+n-s}+\frac{\tilde{c}}{12} \chi_{m n}, \\
{\left[\tilde{L}_{m}, \tilde{G}_{\alpha}^{i}\right] } & =\sum_{s=-g_{0}}^{g_{0}} \mathscr{B}_{m \alpha}^{s} \tilde{G}_{m+n-s}^{i}, \\
{\left[\tilde{L}_{m},\left(J_{L}^{i}\right)_{n}\right] } & =\sum_{s=-g_{0}}^{g_{0}} \mathfrak{R}_{m n}^{s}\left(J_{L}^{i}\right)_{m+n-s}, \\
{\left[\tilde{L}_{m}, \Gamma_{\alpha}\right] } & =\sum_{s=-g_{0}}^{g_{0}} \mathfrak{J}_{m \alpha}^{s} \Gamma_{m+\alpha-s} .
\end{aligned}
$$

The structure constants are relegated to the Appendix. We observe that the modes of the redefined energy momentum tensor satisfies the $\mathrm{K}-\mathrm{N}$ algebra with a modified central charge $\tilde{c}$, where $\tilde{c}=c / 4 \gamma(1-\gamma), c$ being the original central charge. The algebra involving the supercharges and the weight $\frac{1}{2}$ fields are:

$$
\begin{aligned}
\left\{\tilde{G}_{\alpha}^{i}, \tilde{G}_{\beta}^{j}\right\} & =-i \varepsilon^{i j k} \sum_{s=-g_{0}}^{g_{0}} T_{\alpha \beta}^{s}\left(J_{L}^{k}\right)_{\alpha+\beta-s}, \\
{\left[\left(J_{L}^{i}\right)_{m}, \tilde{G}_{\alpha}^{j}\right] } & =i \varepsilon^{i j k} \sum_{s=-g / 2}^{g / 2} \mathscr{D}_{m \alpha}^{s} \tilde{G}_{m+\alpha-s}^{k}, \\
{\left[\left(J_{L}^{i}\right)_{m}, \tilde{G}_{\alpha}^{i}\right] } & =\sum_{s}^{g_{0}} U_{m \alpha}^{s} \Gamma_{m+\alpha-s}, \\
{\left[\Gamma_{\alpha}, \Gamma_{\beta}\right] } & =\frac{2 \tilde{c}}{3} \mathscr{J}_{\alpha \beta}, \\
{\left[\left(J_{L}^{i}\right)_{m}, \Gamma_{\alpha}\right] } & =0, \\
{\left[\Gamma_{\alpha}, \tilde{G}_{\beta}^{i}\right] } & =\sum_{s=-g / 2}^{g / 2} \mathscr{F}_{\alpha \beta}^{s}\left(J_{L}^{i}\right)_{\alpha+\beta-s} .
\end{aligned}
$$

The dimension one current satisfies,

$$
\left[\left(J_{L}^{i}\right)_{m},\left(J_{L}^{i}\right)_{n}\right]=i \varepsilon^{i j k} \sum_{s=-g / 2}^{g / 2} H_{m n}^{s}\left(J_{L}^{k}\right)_{m+n-s}+\frac{\tilde{c}}{3} \delta^{i j} O_{m n} .
$$

The structure constants are presented in the Appendix.

The remaining generators organize themselves into a weight zero primary superfield modulo certain central terms, with respect to the $N=3$ superconformal 
symmetry. These consist of the operators $u(P), \tilde{Q}^{i}(P), J_{R}^{i}(P)$ and $\tilde{G}^{4}(P)$ of weights $0, \frac{1}{2}, 1$ and $\frac{3}{2}$ respectively. Following exactly the same procedure as before we can derive the transformation properties of these operators with respect to the $N=3$ superconformal generators. The explicit form of the algebra may be derived following by the now familiar procedure,

$$
\begin{aligned}
\left\{\tilde{G}_{\alpha}^{i}, \tilde{G}_{\beta}^{4}\right\} & =-\sum_{s=-g_{0}}^{g_{0}} T_{\alpha \beta}^{s}\left(J_{R}^{i}\right)_{\alpha+\beta-s}, \\
{\left[\tilde{G}_{\alpha}^{i},\left(J_{R}^{i}\right)_{m}\right] } & =\sum_{s=-g / 2}^{g / 2} \beta_{m \alpha}^{s} \tilde{G}^{i} 4_{m+\alpha-s}, \\
{\left[\tilde{G}_{\alpha}^{i},\left(J_{R}^{j}\right)_{m}\right] } & =-i \varepsilon^{i j k} \sum_{s=-g_{0}}^{g_{0}} \rho_{m \alpha}^{s} \tilde{Q}_{m+\alpha-s}^{k}, \\
\left\{\tilde{G}_{\alpha}^{i}, \tilde{Q}_{\beta}^{i}\right\} & =\sum_{s=-g_{0}}^{g_{0}} \eta_{\alpha \beta}^{s} u_{\alpha \beta}^{s}+i(2 \gamma-1) \frac{\tilde{c}}{3} \xi_{\alpha \beta}, \\
\left\{\tilde{G}_{\alpha}^{i}, \tilde{Q}_{\beta}^{j}\right\} & =i \varepsilon^{i j k} \sum_{s=-g / 2}^{g / 2} \zeta_{\alpha \beta}^{s}\left(J_{R}^{k}\right)_{\alpha+\beta-s}, \\
{\left[\tilde{G}_{\alpha}^{i}, u_{m}\right] } & =\sum_{s=-g / 2}^{g / 2} \gamma_{m \alpha}^{s} \tilde{Q}_{m+\alpha-s}^{i} .
\end{aligned}
$$

The transformation under the weight 1 generator of the $N=3$ superconformal symmetry may be obtained in the same manner. This gives us,

$$
\begin{aligned}
{\left[\left(J_{L}^{i}\right)_{m}, \tilde{G}_{\alpha}^{4}\right] } & =\sum_{s=-g_{0}}^{g_{0}} \Delta_{m \alpha}^{s} \tilde{Q}_{m+\alpha-s}^{i}, \\
{\left[\left(J_{L}^{i}\right)_{m},\left(J_{R}^{i}\right)_{n}\right] } & =-i(2 \gamma-1) \frac{\tilde{c}}{3} \Theta_{m n}, \\
{\left[\left(J_{L}^{i}\right)_{m},\left(J_{R}^{j}\right)_{n}\right] } & =i \varepsilon^{i j k} \sum_{s=-g / 2}^{g / 2} \Gamma_{m \alpha}^{s}\left(J_{R}^{k}\right)_{m+n-s}, \\
{\left[\left(J_{L}^{i}\right)_{m}, \tilde{Q}_{\alpha}^{j}\right] } & =i \varepsilon^{i j k} \sum_{s=-g / 2}^{g / 2} \Xi_{m \alpha}^{s} \tilde{Q}_{m+\alpha-s}^{k}, \\
{\left[\left(J_{L}^{i}\right)_{m}, \tilde{Q}_{\alpha}^{i}\right] } & =0 .
\end{aligned}
$$

For the weight half generator the transformations are,

$$
\begin{aligned}
{\left[\Gamma_{\alpha}, \tilde{G}_{\beta}^{4}\right] } & =\sum_{s=-g_{0}}^{g_{0}} \Psi_{\alpha \beta}^{s} u_{\alpha+\beta-s}-i \frac{\tilde{c}}{3} \Lambda_{\alpha \beta}, \\
{\left[\Gamma_{\alpha},\left(J_{R}^{i}\right)_{m}\right] } & =\sum_{s=-g / 2}^{g / 2} \mathscr{V}_{m \alpha}^{s} \tilde{Q}_{m+\alpha-s}^{i}, \\
{\left[\Gamma_{\alpha}, \tilde{Q}_{\alpha}^{i}\right]=0 } & =\left[\Gamma_{\alpha}, u_{m}\right] .
\end{aligned}
$$

The structure constants of this algebra are once again relegated to the Appendix.

It may be verified that these relations are the global generalization of the transformation properties of the components of a weight zero superfield under the 
$N=3$ supersymmetry transformations on the complex plane, modulo certain central extensions. This concludes our discussion of the $N=3$ subalgebra on $\Sigma_{g}$. In the next section we twist this theory to extract a TCFT and show that the resulting theory posesses a residual $N=2$ supersymmetry.

\section{Topological Structure}

We have obtained an $N=3 \mathrm{~K}-\mathrm{N}$ subalgebra of the original $N=4 \mathrm{~K}-\mathrm{N}$ algebra in the last section. We now proceed to twist this algebra to obtain the topological version of the $\mathrm{K}-\mathrm{N}$ algebra. To do this we make the following redefinitions of the generators of the $N=3$ superalgebra:

$$
\begin{aligned}
\mathscr{G}^{ \pm}(P) & =\frac{1}{\sqrt{2}}\left[\tilde{G}^{1}(P) \pm \tilde{G}^{2}(P)\right], \\
\mathscr{T}(P) & =\tilde{T}(P)+\frac{1}{2} d_{P} J^{g h}(P), \\
J^{g h}(P) & =\left(J_{L}\right)^{3}(P) .
\end{aligned}
$$

Once again we refrain from explicitly presenting the mode expansions as they are obvious from the identification of the weights of the redefined generators.

Following the procedure of the earlier sections it may be verified that the new generators in the twisted version of the theory satisfy the topological $\mathrm{K}-\mathrm{N}$ algebra outlined in Sect. II. As before the generators $\mathscr{G}^{ \pm}(P)$ transform as a dimension one BRST current and a dimension two BRST partner of $\mathscr{T}(P)$. The BRST charge is given as before:

$$
Q_{B}=\oint_{C_{\tau}} \mathscr{G}^{+}(P)=\mathscr{G}_{g / 2}^{+} .
$$

Consequently $J^{\text {gh }}(P)$ is identified as the ghost number current of the TCFT on $\Sigma_{g}$. We relabel the operators as $\mathscr{G}^{+}(P)=Q(P)$ and $\mathscr{G}^{-}(P)=G(P) \cdot Q(P)$ and $G(P)$ are the new weight 1 and weight 2 operators.

We proceed now to show that the topological field theory on $\Sigma_{g}$ which realizes the topological $\mathrm{K}-\mathrm{N}$ algebra posesses a residual $N=2$ supersymmetry. The remaining generators organize themselves into a weight zero superfield of this $N=2$ supersymmetry. To obtain the $N=2$ superconformal structure of the TCFT on $\Sigma_{g}$ we start off by redefining some of the generators in the following fashion:

$$
\begin{aligned}
\mathscr{G}^{3}(P) & =\tilde{G}^{3}(P)-\mathrm{id}_{P} Q^{4}(P), \\
\mathscr{G}^{4}(P) & =\tilde{G}^{4}(P)+\mathrm{id}_{P} Q^{3}(P), \\
\left(J_{L}^{ \pm}\right)(P) & = \pm \frac{1}{\sqrt{2}}\left[J_{L}^{1}(P) \pm i J_{L}^{2}(P)\right], \\
\left(J_{R}^{ \pm}\right)(P) & = \pm \frac{1}{\sqrt{2}}\left[J_{R}^{1}(P) \pm i J_{R}^{2}(P)\right], \\
\left(\tilde{J}_{R}^{3}\right)(P) & =i\left[D^{+3}(P)-D^{-3}(P)\right]+U(P), \\
Q^{ \pm}(P) & =\frac{1}{\sqrt{2}}\left[\tilde{Q}^{1}(P) \pm \tilde{Q}^{2}(P)\right] .
\end{aligned}
$$


As before we can go over to the local expressions of these operators and obtain their modified OPE's with the $N=2$ superconformal generators. From these OPE's it is seen that the supercharge $\mathscr{G}^{3}(P)$ transforms as a weight $\frac{3}{2}$ operator and the operators $\left(J_{L}^{ \pm}\right)(P)$ and $\left(J_{R}^{ \pm}\right)(P)$ in the twisted theory transform as weight $\frac{1}{2}$ and $\frac{3}{2}$ fields respectively. Furthermore the weights of the various other fields are also shifted accordingly. $\mathscr{G}^{4}(P),\left(\tilde{J}_{R}^{3}\right)(P)$ and $\tilde{Q}^{ \pm}(P)$ transform as weight $\frac{3}{2}, 1,0$ and 1 respectively. We rename the operators in the twisted theory as follows:

$$
\begin{gathered}
\left(J_{L}^{ \pm}\right)(P)=\tilde{J}_{L}^{ \pm}(P), \\
\left(J_{R}^{ \pm}\right)(P)=\tilde{J}_{\bar{R}}^{ \pm}(P), \\
Q^{ \pm}(P)=\tilde{Q}^{ \pm}(P) .
\end{gathered}
$$

The generators may now be expanded as earlier in the appropriate $\mathrm{K}-\mathrm{N}$ bases and then inverted to obtain the relevant Fourier projections. The algebra may now be obtained explicitly following the method of the earlier sections. We see that the operators $\mathscr{G}^{3}, \mathscr{G}^{4},\left(\widetilde{J}_{R}^{3}\right)$, and $\mathscr{L}$ satisfy an $N=2$ super $\mathrm{K}-\mathrm{N}$ algebra on $\Sigma_{g}$.

$$
\begin{aligned}
{\left[\mathscr{L}_{m}, \mathscr{G}_{\alpha}^{3}\right] } & =\sum_{s=-g_{0}}^{g_{0}} \mathscr{B}_{m \alpha}^{s} \mathscr{G}_{m+\alpha-s}^{3}, \\
\left\{\mathscr{G}_{\alpha}^{i}, \mathscr{G}_{\beta}^{i}\right\} & =\sum_{s=-g / 2}^{g / 2} F_{\alpha \beta}^{s} \mathscr{L}_{\alpha+\beta-s} \quad i=3,4, \\
\left\{\mathscr{G}_{\alpha}^{3}, \mathscr{G}_{\beta}^{3}\right\} & =i \sum_{s=-g / 2}^{g / 2} \mathscr{E}_{\alpha \beta}^{s}\left(\tilde{J}_{R}^{3}\right)_{\alpha+\beta-s}, \\
{\left[\left(\tilde{J}_{R}^{3}\right)_{m}, \mathscr{G}_{\alpha}^{3}\right] } & =i \sum_{s=-g / 2}^{g / 2} \mathscr{D}_{m \alpha}^{s} \mathscr{G}_{m+\alpha-s}^{3}, \\
{\left[\left(\tilde{J}_{R}^{3}\right)_{m}, \mathscr{G}_{\alpha}^{4}\right] } & =-i \sum_{s=-g / 2}^{g / 2} \mathscr{D}_{m \alpha}^{s} \mathscr{G}_{m+\alpha-s}^{4} .
\end{aligned}
$$

This completes our derivation of the $N=2$ superconformal structure of the TCFT associated with the topological $\mathrm{K}-\mathrm{N}$ algebra obtained from twisting the $N=3$ subalgebra of the $N=4$ super $\mathrm{K}-\mathrm{N}$ algebra.

We now proceed to discuss the weight zero primary superfield of this $N=2$ superconformal symmetry. The components of this superfield are the four generators $u(P), \Gamma(P), \widetilde{Q}^{3}(P)$ and $J^{\mathrm{gh}}(P)$. The primary nature is obvious from the transformation under the conformal generator $\mathscr{L}_{m}$. The transformation under the other $N=2$ superconformal generators may be derived following the standard procedure as outlined earlier. For the weight $\frac{3}{2}$ generators we have

$$
\begin{aligned}
& {\left[\mathscr{G}_{\alpha}^{3}, J_{m}^{\mathrm{gh}}\right]=\sum_{s=-g_{0}}^{g_{0}} \mathscr{A}_{m \alpha}^{s} \Gamma_{m+n-s},} \\
& {\left[\mathscr{G}_{\alpha}^{4}, J_{m}^{\mathrm{gh}}\right]=\sum_{s=-g_{0}}^{g_{0}} \mathscr{A}_{m \alpha}^{s} \tilde{Q}_{m+\alpha-s}^{3},}
\end{aligned}
$$




$$
\begin{aligned}
\left\{\mathscr{G}_{\alpha}^{3}, \tilde{Q}_{\beta}^{3}\right\} & =\frac{1}{2} \sum_{s=-g_{0}}^{g_{0}} \mu_{\alpha \beta}^{s} u_{\alpha+\beta-s} \\
& =-\left\{\mathscr{G}_{\alpha}^{4}, \tilde{Q}_{\beta}^{3}\right\}, \\
{\left[\mathscr{G}_{\alpha}^{3}, u_{m}\right] } & =\sum_{s=-g / 2}^{g / 2} \mathscr{P}_{m \alpha}^{s} \tilde{Q}_{m+\alpha-s}^{3}, \\
{\left[\mathscr{G}_{\alpha}^{4}, u_{m}\right] } & =\sum_{s=-g / 2}^{g / 2} \mathscr{P}_{m \alpha}^{s} \Gamma_{m+\alpha-s} .
\end{aligned}
$$

The transformation with respect to the $S U(2)$ generator is derived in a similar fashion to give

$$
\begin{aligned}
& {\left[\left(\tilde{J}_{R}^{3}\right)_{m}, \Gamma_{\alpha}\right]=i \sum_{s=-g / 2}^{g / 2} \mathscr{R}_{m \alpha}^{s} \tilde{Q}_{m+\alpha-s}^{3},} \\
& {\left[\left(\tilde{J}_{R}^{3}\right)_{m}, \tilde{Q}_{\alpha}^{3}\right]=-i \sum_{s=-g / 2}^{g / 2} \mathscr{R}_{m \alpha}^{s} \Gamma_{m+\alpha-s},} \\
& {\left[\left(\tilde{J}_{R}^{3}\right)_{m}, J_{n}^{g h}\right]=\frac{\tilde{c}}{3}(2 \gamma-1) \mathscr{Y}_{m n},} \\
& {\left[\left(\tilde{J}_{R}^{3}\right)_{m}, u_{n}\right]=-\frac{2 i}{3} \tilde{c} \vartheta_{m n} .}
\end{aligned}
$$

It may be verified that these transformation laws are the global generalization of the similar laws of the weight zero $N=2$ superfield on the complex plane. This can be most suitably observed by evaluating the above expressions on a local co-ordinate patch around the punctures. The structure constants for the above algebra are presented explicitly in the Appendix. This completes our discussion of the $N=2$ superconformal structure of the TCFT on $\Sigma_{g}$ associated with the topological $\mathrm{K}-\mathrm{N}$ algebra derived at the beginning of this section. In the next section we present the explicit BRST structure of the full theory.

\section{BRST Structure of the Topological Field Theory}

In this section we explicitly elucidate the BRST structure of the $N=2$ TSCFT. We observe that each generator is the generalized BRST derivative of some other generators, leading to a BRST partnership of all the generators characteristic of any topological field theory. Here we only illustrate the BRST partnership of those generators which are not part of the set of generators of the $N=2$ SCFT. For these the BRST partnership is standard. For the rest of the generators we obtain the BRST derivative conditions by obtaining their BRST transformations. Thus we have the following algebra:

$$
\begin{aligned}
{\left[Q_{n},\left(\tilde{J}_{L}^{-}\right)_{\alpha}\right] } & =\sum_{s=-g_{0}}^{g_{0}} \mathscr{S}_{n \alpha}^{s} \Gamma_{n+\alpha-s}-\sum_{s=-g / 2}^{g / 2} \mathscr{H}_{n \alpha}^{s} \mathscr{G}_{n+\alpha-s}^{3}, \\
{\left[Q_{n}, \mathscr{G}_{\alpha}^{3}\right] } & =\sum_{s=-g_{0}}^{g_{0}} \mathscr{U}_{m \alpha}^{s}\left(\tilde{J}_{L}^{+}\right)_{n+\alpha-s}
\end{aligned}
$$




$$
\begin{aligned}
{\left[Q_{n}, \Gamma_{\alpha}\right] } & =\sum_{s=-g / 2}^{g / 2} \mathscr{M}_{n \alpha}^{s}\left(\tilde{J}_{L}^{+}\right)_{n+\alpha-s}, \\
{\left[Q_{n}, \tilde{Q}_{\alpha}^{3}\right] } & =-\sum_{s=-g / 2}^{g / 2} \mathscr{M}_{n \alpha}^{s}\left(\tilde{J}_{R}^{+}\right)_{n+\alpha-s}, \\
{\left[Q_{n}, u_{m}\right] } & =\sum_{s=-g / 2}^{g / 2} \mathscr{W}_{m n}^{s}\left(\tilde{J}_{R}^{+}\right)_{n+\alpha-s}, \\
{\left[Q_{n},\left(\tilde{J}_{R}^{-}\right)_{\alpha}\right] } & =\sum_{s=-g_{0}}^{g_{0}} \mathscr{X}_{n \alpha}^{s} \tilde{Q}_{n+\alpha-s}^{3}+\sum_{s=-g / 2}^{g / 2} v_{n \alpha}^{s} \mathscr{G}_{n+\alpha-s}^{4}, \\
{\left[Q_{n}, \mathscr{G}_{\alpha}^{4}\right] } & =\sum_{s=-g_{0}}^{g_{0}} Y^{n \alpha}\left(\tilde{J}_{R}^{+}\right)_{n+\alpha-s}, \\
{\left[Q_{n}, \tilde{Q}_{m}^{-}\right] } & =\sum_{s=-g / 2}^{g / 2} \mathscr{N}_{n m}^{s}\left(\tilde{J}_{R}^{3}\right)_{m+n-s}-i \frac{c}{3}(2 \gamma-1) \mathscr{Z}_{n m},
\end{aligned}
$$

where $Q(z)$ is the weight one BRST current for the $N=2$ TSCFT. The structure constants of the algebra are relegated to Appendix.

The BRST structure may now be obtained from Eqs. (6.1-6.8) by setting $n=g / 2$. Recall from Sects. II and V that for $n=g / 2$ we have $Q_{g / 2}=Q_{B}$ where $Q_{B}$ is the BRST charge. Notice from Appendix that the structure constants $\mathscr{S}_{n \alpha}^{s}, \mathscr{X}_{n \alpha}^{s}, \mathscr{Z}_{n m}$ and $Y_{n \alpha}^{s}$ are all zero as their expressions contain $d_{P} A_{g / 2}(P)=0$ as $A_{g / 2}(P)=1$ from Eq. (2.4).

However in contrast to the case on the complex plane where the algebra closes onto a single mode of the BRST partner of the relevant generator, on $\Sigma_{g}$ it closes to a linear combination of the modes. This is a global generalization of the BRST derivative condition on a Riemann surface. So we may remark that on $\Sigma_{g}$, each generator is a generalized BRST derivative of some other generator. We may recover the complex plane results by going to a local co-ordinate system. This concludes our discussion of the full BRST structure of the $N=2$ TSCFT.

\section{Summary and Conclusion}

We have investigated the algebraic structure of a topological superconformal field theory on a compact Riemann surface of arbitrary genus. In this context we have used the $\mathrm{K}-\mathrm{N}$ global operator formalism to define an $N=4$ super $\mathrm{K}-\mathrm{N}$ algebra on a Riemann surface. Subsequently we have extracted an $N=3$ super $\mathrm{K}-\mathrm{N}$ algebra from it via certain suitable redefinitions of the generators. It was found that the remaining generators transform as a weight zero primary superfield of this $N=3$ superconformal symmetry. A topological version of the $\mathrm{K}-\mathrm{N}$ algebra was constructed by twisting this $N=3$ algebra and it was shown that there exists a residual $N=2$ superconformal structure. In addition we have described the full BRST structure of the theory and have identified the BRST partners of all the generators. We have observed that on the Riemann surface the BRST derivative condition on the complex plane is modified to a generalized BRST derivative condition which reduces to the complex plane results $[2,4]$ when evaluated in a local co-ordinate patch. The BRST charge in the formalism developed is genus dependent. Consequently the cohomology of the physical states in the Hilbert space of the associated 
TSCFT should also be genus dependent in accordance with the results of [16] for a TCFT on a Riemann surface.

In conclusion we remark that the $\mathrm{K}-\mathrm{N}$ operator formalism provides an elegant and simple method to probe the algebraic structure of TCFT and TSCFT on a Riemann surface. Our investigations reveal an interesting global generalization of the BRST charge and the BRST derivative conditions for the generators.

It would be an interesting exercise to extend this analysis to the free field realization of these TCFT and two dimensional topological gravity. Investigation into the structure of the minimal TCFT coupled to topological gravity in this framework would provide the construction of a topological string theory on a Riemann surface. Work in this direction is in progress.

Acknowledgements. All of us would like to thank Samir K. Paul for numerous discussions regarding K-N algebra. We would also like to thank Vijay Agrawal, V.S. Uma and Suresh. K. Patra for help in preparation of the manuscript.

\section{Appendix}

In this appendix we present the structure constants appearing in the various algebras in the text. We express these in their global forms valid everywhere on the Riemann surface by using

$$
f^{\lambda}(P)=\Phi(z)(d z)^{\lambda}
$$

Here $f^{\lambda}$ is a meromorphic form on $\Sigma_{g}$ and $\Phi(z)$ stands for the components in some local co-ordinate system $z$,

$$
\begin{aligned}
A_{m n}^{s} & =\frac{1}{2 \pi i} \oint_{P \in C_{\tau}}\left[d_{P} e_{m}(P) e_{n}(P)-e_{m} d_{P} e_{n}(P)\right] \Omega_{m+n-s} \\
C_{m n}^{s} & =\frac{1}{2 \pi i} \oint_{P \in C_{\tau}}\left[d_{P} A_{n}(P)\right] e_{m}(P) W_{m+n-s}(P) \\
P_{m n}^{s} & =\frac{2}{2 \pi i} \oint_{P \in C_{\tau}} A_{n}(P) e_{m}(P) \Omega_{m+n-s}(P) \\
E_{m n}^{s} & =\frac{2}{2 \pi i} \oint_{P \in C_{\tau}} e_{m}(P) d_{P} A_{n}(P) W_{m+n-s}(P) \\
\Pi_{m n} & =\frac{1}{2 \pi i} \oint_{P \in C_{\tau}} e_{m}(P) d_{P}^{2} A_{n}(P) W_{m+n-s}(P) \\
t_{j m} & =\frac{1}{2 \pi i} \oint_{P \in C_{\tau}} d_{P} A_{j}(P) e_{m}(P) \\
K_{j n} & =\frac{1}{2 \pi i} \oint_{P \in C_{\tau}} A_{n}(P) d_{P} A_{j}(P) \\
D_{m n}^{s} & =-\frac{1}{2 \pi i} \oint_{P \in C_{\tau}} A_{m}(P) e_{n}(P) \Omega_{m+n-s}(P) \\
B_{m n}^{s} & =\frac{1}{2 \pi i} \oint_{P \in C_{\tau}} A_{m}(P) A_{n}(P) W_{m+n-s}(P)
\end{aligned}
$$


The global expressions for the structure constants of the $N=4$ super $\mathrm{K}-\mathrm{N}$ algebra on the Riemann surface $\Sigma_{g}$ Eqs. (3.11-3.26) in Sect. III is given as,

$$
\begin{aligned}
& V_{m \alpha}^{s}=\frac{1}{2 \pi i} \oint_{P \in C_{\tau}} C_{\alpha}(P)\left[\frac{3}{2} d_{P} e_{m}(P) B_{m+\alpha-s}(P)+e_{m}(P) d_{P} B_{m+\alpha-s}(P)\right], \\
& X_{m \alpha}^{s}=\frac{1}{2 \pi i} \oint_{P \in C_{\mathrm{r}}} g_{-\alpha}(P)\left[\frac{1}{2} d_{P} e_{m}(P) g_{m+\alpha-s}+e_{m}(P) d_{P} g_{m+\alpha-s}(P)\right], \\
& \mathscr{E}_{\alpha \beta}^{s}=\frac{1}{2 \pi i} \oint_{P \in C_{\tau}}\left[2 C_{\beta}(P) d_{P} C_{\alpha}(P) W_{\alpha+\beta-s}(P)\right. \\
& \left.+C_{\beta}(P) C_{\alpha}(P) d_{P} W_{\alpha+\beta-s}(P)\right] \text {, } \\
& F_{\alpha \beta}^{s}=\frac{1}{2 \pi i} \oint_{P \in C_{\tau}} C_{\beta}(P) d_{P}^{2} C_{\alpha}(P) \Omega_{\alpha+\beta-s}(P), \\
& \mathscr{Q}_{\alpha \beta}=\frac{1}{2 \pi i} \underset{P \in C_{\tau}}{\oint} C_{\beta}(P) d_{P}^{2} C_{\alpha}(P), \\
& Y_{m \alpha}^{s}=\frac{1}{2 \pi i} \underset{P \in C_{\tau}}{\oint} C_{\alpha}(P) A_{m}(P) B_{m+\alpha-s}(P), \\
& Z_{m \alpha}^{s}=\frac{1}{2 \pi i} \oint_{P \in C_{\tau}} C_{\alpha}(P) d_{P} A_{m}(P) B_{m+\alpha-s}(P), \\
& I_{m n}^{s}=\frac{1}{2 \pi i} \oint_{P \in C_{\mathrm{r}}} A_{m}(P) A_{n}(P) W_{m+n-s}(P), \\
& J_{m n}=\frac{1}{2 \pi i} \underset{P \in C_{\tau}}{\oint} A_{n}(P) d_{P} A_{m}(P), \\
& \mathscr{K}_{\alpha \beta}^{s}=\frac{1}{2 \pi i} \underset{P \in C_{\tau}}{\oint} C_{\beta}(P) G_{-\alpha}(P) W_{\alpha+\beta-s}(P), \\
& \mathscr{L}_{m \alpha}^{s}=\frac{1}{2 \pi i} \oint_{P \in C_{\tau}} g_{-\alpha}(P) A_{m}(P) g_{m+\alpha-s}(P), \\
& N_{m \alpha}^{s}=\frac{1}{2 \pi i} \oint_{P \in C_{\tau}} C_{\alpha}(P) d_{P} A_{m}(P) g_{m+\alpha-s}(P), \\
& \mathscr{P}_{\alpha \beta}=\frac{1}{2 \pi i} \underset{P \in C_{\tau}}{\oint} g_{-\beta}(P) g_{-\alpha}(P),
\end{aligned}
$$

with the only non zero elements of $\alpha^{ \pm i}$ being, $\alpha_{j k}^{ \pm i}=\frac{1}{2} \varepsilon_{i j k}$ and $\alpha_{j 4}^{ \pm i}=\alpha_{4 j}^{ \pm i}=\frac{1}{2} \delta_{i j}$.

The structure constants of the $N=3 \mathrm{~K}-\mathrm{N}$ subalgebra of the $N=4 \mathrm{~K}-\mathrm{N}$ superalgebra on $\Sigma_{g}$ Eqs. (4.9-4.17) in Sect. IV, are given in the global form as follows:

$$
\begin{aligned}
& \mathscr{B}_{m \alpha}^{s}=\frac{1}{2 \pi i} \oint_{P \in C_{\tau}}\left[\frac{1}{2} C_{\alpha}(P) d_{P} e_{m}(P)-d_{P} C_{\alpha}(P) e_{m}(P)\right] B_{i+j-s}(P), \\
& \mathfrak{R}_{m n}^{s}=-\frac{1}{2 \pi i} \oint_{P \in C_{\tau}} e_{m}(P) d_{P} A_{n}(P) W_{m+n-s}(P),
\end{aligned}
$$




$$
\begin{aligned}
\mathfrak{J}_{m \alpha}^{s} & =-\frac{1}{2 \pi i} \oint_{P \in C_{\tau}} e_{m}(P) d_{P} g_{-\alpha}(P) g_{m+\alpha-s}(P), \\
T_{\alpha \beta}^{s} & =\frac{1}{2 \pi i} \oint_{P \in C_{\tau}} d_{P} C_{\beta}(P) C_{\alpha}(P) W_{\alpha+\beta-s}(P), \\
\mathscr{D}_{m \alpha}^{s} & =\frac{1}{2 \pi i} \oint_{P \in C_{\tau}} A_{m}(P) C_{\alpha}(P) B_{m+\alpha-s}(P), \\
U_{m \alpha}^{s} & =\frac{1}{2 \pi i} \oint_{P \in C_{\tau}} C_{\alpha}(P) d_{P} A_{m}(P) g_{m+\alpha-s}(P), \\
H_{m n}^{s} & =\frac{1}{2 \pi i} \oint_{P \in C_{\tau}} A_{n}(P) A_{m}(P) W_{m+n-s}(P), \\
O_{m n} & =\frac{1}{2 \pi i} \oint_{P \in C_{\tau}} A_{n}(P) d_{P} A_{m}(P), \\
\mathscr{J}_{\alpha \beta} & =\frac{1}{2 \pi i} \oint_{P \in C_{\tau}} g_{-\alpha}(P) g_{-\beta}(P), \\
\mathscr{F}_{\alpha \beta}^{s} & =\frac{1}{2 \pi i} \oint_{P \in C_{\tau}} g_{-\alpha}(P) C_{\beta}(P) W_{\alpha+\beta-s} .
\end{aligned}
$$

We also present the structure constants for the algebra of the transformations of the components of the residual weight zero superfield under the $N=3$ superconformal transformations in Eqs. (4.18-4.31),

$$
\begin{aligned}
\beta_{m \alpha}^{s} & =\frac{1}{2 \pi i} \oint_{P \in C_{\tau}} A_{m}(P) C_{\alpha}(P) B_{m+\alpha-s}(P) \\
\rho_{m \alpha}^{s} & =\frac{1}{2 \pi i} \oint_{P \in C_{\tau}} A_{m}(P) d_{P} C_{\alpha}(P) g_{m+\alpha-s}(P), \\
\eta_{\alpha \beta}^{s} & =\frac{1}{2 \pi i} \oint_{P \in C_{\tau}} g_{-\beta}(P) C_{\alpha}(P) d_{P} A_{\alpha+\beta-s}(P), \\
\xi_{\alpha \beta} & =\frac{1}{2 \pi i} \oint_{P \in C_{\tau}} g_{-\beta}(P) d_{P} C_{\alpha}(P) \\
\zeta_{\alpha \beta}^{s} & =\frac{1}{2 \pi i} \oint_{P \in C_{\tau}} g_{-\beta}(P) C_{\alpha}(P) W_{\alpha+\beta-s}(P) \\
\gamma_{m \alpha}^{s} & =\frac{1}{2 \pi i} \oint_{P \in C_{\tau}} W_{m}(P) C_{\alpha}(P) g_{\alpha+m-s}(P), \\
\Delta_{m \alpha}^{s} & =\frac{1}{2 \pi i} \oint_{P \in C_{\tau}} d_{P} A_{m}(P) C_{\alpha}(P) g_{m+\alpha-s}(P), \\
\Theta_{m n} & =\frac{1}{2 \pi i} \oint_{P \in C_{\tau}} A_{n}(P) d_{P} A_{m}(P)
\end{aligned}
$$




$$
\begin{aligned}
& \Gamma_{m \alpha}^{s}=\frac{1}{2 \pi i} \oint_{P \in C_{\tau}} A_{n}(P) A_{m}(P) W_{m+n-s}(P), \\
& \Xi_{m \alpha}^{s}=\frac{1}{2 \pi i} \oint_{P \in C_{\tau}} A_{m}(P) g_{-\alpha}(P) g_{m+\alpha-s}(P), \\
& \Psi_{\alpha \beta}^{s}=-\frac{1}{2} \frac{1}{2 \pi i} \oint_{P \in C_{\tau}} C_{\beta}(P) g_{-\alpha}(P) d_{P} A_{\alpha+\beta-s}(P), \\
& \Lambda_{\alpha \beta}=-\frac{1}{2 \pi i} \oint_{P \in C_{\tau}} C_{\beta}(P) d_{P} g_{-\alpha}(P), \\
& \mathscr{V}_{m \alpha}^{s}=\frac{1}{2 \pi i} \oint_{P \in C_{\tau}} A_{m}(P) g_{-\alpha}(P) g_{m+\alpha-s}(P) .
\end{aligned}
$$

We now present the explicit global forms for the structure constants of the algebra of transformation of the components of the weight zero primary superfield of the $N=2$ superconformal symmetry in Eqs. (5.19-5.28),

$$
\begin{aligned}
& \mathscr{A}_{m \alpha}^{s}=\frac{1}{2 \pi i} \oint_{P \in C_{\tau}} C_{\alpha}(P)\left[A_{m}(P) d_{P} g_{m+\alpha-s}-g_{m+\alpha-s}(P) d_{P} A_{m}(P)\right], \\
& \mu_{\alpha \beta}^{s}=\frac{1}{2 \pi i} \oint_{P \in C_{\tau}} g_{-\beta}(P) C_{\alpha}(P) d_{P} A_{\alpha+\beta+s}(P), \\
& \mathscr{P}_{m \alpha}^{s}=\frac{1}{2 \pi i} \oint_{P \in C_{\tau}} A_{m}(P) C_{\alpha}(P) g_{m+\alpha-s}(P), \\
& \mathscr{R}_{m \alpha}^{s}=\frac{1}{2 \pi i} \oint_{P \in C_{\tau}} g_{-\alpha}(P) A_{m}(P) g_{m+\alpha-s}(P), \\
& \mathscr{Y}_{m n}=\frac{1}{2 \pi i} \oint_{P \in C_{\tau}} A_{n}(P) d_{P} A_{m}(P), \\
& \vartheta_{m n}=\frac{1}{2 \pi i} \oint_{P \in C_{\tau}} W_{n}(P) d_{P} A_{m}(P) .
\end{aligned}
$$

Having presented the OPEs we now give the explicit global expression for the structure constants of the algebra in Eqs. (6.1-6.8) for the transformation under the weight one BRST current the integral of which over a level curve $C_{\tau}$ defines the BRST charge,

$$
\begin{aligned}
& \mathscr{S}_{n \alpha}^{s}=\frac{1}{2 \pi i} \oint_{P \in C_{\tau}} C_{\alpha}(P) d_{P} A_{n}(P) g_{n+\alpha-s}(P), \\
& \mathscr{H}_{n \alpha}^{s}=\frac{1}{2 \pi i} \oint_{P \in C_{\tau}} C_{\alpha}(P) A_{n}(P) B_{n+\alpha-s}(P),
\end{aligned}
$$




$$
\begin{aligned}
\mathscr{U}_{m \alpha}^{s} & =\frac{1}{2 \pi i} \oint_{P \in C_{\tau}} C_{\alpha}(P) d_{P} A_{m}(P) g_{m+\alpha-s}(P), \\
\mathscr{M}_{n \alpha}^{s} & =\frac{1}{2 \pi i} \oint_{P \in C_{\tau}} g_{\alpha}(P) A_{n}(P) g_{n+\alpha-s}(P), \\
\mathscr{W}_{m n}^{s} & =2 \frac{1}{2 \pi i} \oint_{P \in C_{\tau}} W_{m}(P) A_{n}(P) A_{n+m-s}(P), \\
\mathscr{X}_{n \alpha}^{s} & =\frac{1}{2 \pi i} \oint_{P \in C_{\tau}} C_{\alpha}(P) d_{P} A_{n}(P) g_{\alpha+n-s}(P), \\
v_{n \alpha}^{s} & =\frac{1}{2 \pi i} \oint_{P \in C_{\tau}} C_{\alpha}(P) A_{n}(P) B_{n+\alpha-s}(P), \\
\mathscr{F}_{n \alpha}^{s} & =-\frac{1}{2 \pi i} \oint_{P \in C_{\tau}} C_{\alpha}(P) d_{P} A_{n}(P) g_{n+\alpha-s}(P), \\
\mathscr{N}_{n m}^{s} & =i \frac{1}{2 \pi i} \oint_{P \in C_{\tau}} A_{n}(P) A_{m}(P) W_{m+n-s}(P), \\
\mathscr{Z}_{n m} & =\frac{1}{2 \pi i} \oint_{P \in C_{\tau}} A_{m}(P) d_{P} A_{n}(P) .
\end{aligned}
$$

For $n=g / 2$ we have the global form of the BRST derivative conditions. Recall that from Sect. II we have the BRST charge as $Q_{B}=Q_{g / 2}$.

\section{References}

1. Witten, E.: Commun. Math. Phys. 117, 353 (1988); 118, 411 (1988)

2. Eguchi, T., Yang, S.K.: Mod. Phys. Lett. A5, 1693 (1990)

3. Ademollo, M. et al.: Phys. Lett. B62, 105 (1976); Nucl. Phys. B111, 77 (1976); Lerche, W., Vafa, C., Warner, N.: Nucl. Phys. B234, 427 (1989); Di Vecchia, P., Petersen, J., Zheng, H.: Phys. Lett. B162, 327 (1985); Phys. Lett. B174, 280 (1986)

4. Dijkgraaf, R., Verlinde, H., Verlinde, E.: Nucl. Phys. B352, 59 (1991); Li, K.: Nucl. Phys. B354, $711(1991)$

5. Labastida, J.M.F., Pernici, M., Witten, E.: Nucl. Phys. B310, 611 (1988); Montano, D., Sonnenschein, J.: Nucl. Phys. B324, 348 (1989); Verlinde, E., Verlinde, H.: Nucl. Phys. B348, 457 (1991); Myers, R.C., Periwal, V.: Nucl. Phys. B333, 536 (1990)

6. Brézin, E., Kazakov, V.: Phys. Lett. B236, 144 (1990); Gross, D.J., Migdal, A.: Phys. Rev. Lett. 64, 127 (1990); Douglas, M., Shenker, S.: Nucl. Phys. B335, 635 (1990); Douglas, M.: Phys. Lett. 238B, 279 (1990)

7. Witten, E.: Nucl. Phys. B340, 281 (1990); Dijkgraaf, R., Witten, E.: Nucl. Phys. B342, 486 (1990); Distler, J.: Nucl. Phys. B342, 523 (1990); Vafa, C.: Mod. Phys. Lett. A6, 337 (1991)

8. Hughes, J., Li, K.: Phys. Lett. B261, 269 (1991); Cordes, S., O'Connor, M.: Report No. NUB-3017 (1991); Distler, J., Nelson, P.: Phys. Rev. Lett. 66, 1955 (1991)

9. Kumar, A., Maharana, J.: Nucl. Phys. B373, 281 (1992)

10. Kumar A., Chang, D.: Phys. Lett. B193, 181 (1987); Schwimmer, A., Seiberg, N.: Phys. Lett. B184, 191 (1987)

11. Ali, A., Jatkar, D., Kumar, A.: Institute of Physics (Bhubaneswar), Report No. IP/BBSR/ 91-14 
12. Sevrin, A., Troost, W., Van Proeyen, A.: Phys. Lett. B208, 447 (1988); Nucl. Phys. B311, 465 (1987)

13. Krichever, I.M., Novikov, S.P.: Funkts. Anal. i Pril., 21, 46 (1987); 21, 47 (1987); Vadim Knizhnik Memorial Volume, Brink, L., Friedan, D., Polyakov, A.M. (eds.) Singapore: World Scientific 1990.

14. Alberty, J., Taormina, A., Van Baal, P.: Commun. Math. Phys. 120, 249 (1988); Bonora, L., Martellini, M., Rinaldi, M., Russo, J.: Phys. Lett. B206, 444 (1989); Bonora, L., Lugo, A., Matone, M., Russo, J.: Commun. Math. Phys. 123, 329 (1989)

15. Maharana, J., Paul, S.K., Sengupta, G.: Commun. Math. Phys. 139, 527 (1991)

16. Kumar, A., Maharana, J., Sengupta, G.: Lett. Math. Phys. 23, 271 (1991)

17. Xu, K.W., Zhao, Z.Y.: Phys. Rev. D42, 3433 (1990)

Communicated by K. Gawedzki 
